\title{
Icaritin promotes the osteogenesis of bone marrow mesenchymal stem cells via the regulation of sclerostin expression
}

\author{
QIUSHI WEI ${ }^{1,2^{*}}$, BIN WANG ${ }^{3,4^{*}}$, HAILAN HU $^{3}$, CHUHAI XIE $^{3}$, \\ LONG LING $^{3}$, JIANLIANG GAO ${ }^{3}$ and YANMING CAO ${ }^{3}$
}

\begin{abstract}
${ }^{1}$ Hip Preserving Ward, No. 3 Orthopedic Region, The First Affiliated Hospital of Guangzhou University of Chinese Medicine, Guangzhou, Guangdong 510407; ${ }^{2}$ Institute of Hip Diseases, Guangzhou University of Chinese Medicine, Guangzhou, Guangdong 510405; ${ }^{3}$ Department of Orthopedics, The Second Affiliated Hospital of Guangzhou Medical University, Guangzhou, Guangdong 510260; ${ }^{4}$ Department of Orthopedics, People's Hospital of Sanshui, Foshan, Guangdong 528100, P.R. China
\end{abstract}

Received November 21, 2018; Accepted September 30, 2019

DOI: $10.3892 /$ ijmm.2020.4470

\begin{abstract}
Icaritin, a metabolite of icariin, is a potent promoter of bone marrow-derived mesenchymal stem cells (BMSCs) osteogenesis, but the underlying mechanisms remain unclear. To examine the effects of icaritin on osteogenic differentiation, BMSCs were exposed to osteogenic induction medium with or without icaritin pretreatment in the present study. It was identified that icaritin $(0.01-1 \mu \mathrm{M})$ exhibited no cytotoxicity on the proliferative abilities of the BMSCs. Icaritin at $1 \mu \mathrm{M}$ increased alkaline phosphatase activity, mineral deposition and osteoblast-specific gene expression. Treatment with $1 \mu \mathrm{M}$ Icaritin upregulated osteocalcin, RUNX family transcription factor 2 , tissue-nonspecific alkaline phosphatase and $\beta$-catenin, and suppressed sclerostin (SOST) gene expression in different stages of osteogenic differentiation. It was also demonstrated that SOST overexpression inhibited icaritin-induced osteogenesis. The western blot analysis data suggested that ICI 182780, which causes estrogen receptor $\alpha(\mathrm{ER} \alpha)$ degradation, reversed the icaritin-induced decrease in SOST expression, which was inconsistent with the results of immunofluorescence analysis. In conclusion, icaritin was demonstrated to promote the osteogenesis of hBMSCs by downregulating SOST expression, and icaritin-induced suppression of SOST was regulated in part via the $\mathrm{Wnt} / \beta$-catenin/ER $\alpha$ axis.
\end{abstract}

Correspondence to: Dr Yanming Cao, Department of Orthopedics, The Second Affiliated Hospital of Guangzhou Medical University, 250 East Changgang Road, Guangzhou, Guangdong 510260, P.R. China

E-mail: 13729813888@126.com

*Contributed equally

Key words: icaritin, osteogenic differentiation, human bone marrow mesenchymal stem cells, sclerostin expression

\section{Introduction}

Due to their self-renewal capabilities, multi-directional differentiation potential and the fact that they are easily accessible, bone marrow-derived mesenchymal stem cells (BMSCs) have been applied in tissue engineering, especially in the construction of bone defects models (1). As bone defects such as osteoporosis or osteonecrosis of femoral head are primarily due to the decreased osteogenic differentiation of BMSCs (2), it is crucial to develop strategies to promote the osteogenic differentiation of BMSCs in a clinical setting (3).

Sclerostin (SOST), the protein product of the SOST gene, serves a key role in inhibiting osteoblast activity (4). It is also well-known that SOST negatively regulates bone formation through antagonizing the Wnt/ $\beta$-catenin pathway (5). In addition, knockdown of the $S O S T$ gene in mice results in a high bone mass phenotype and resistance to bone loss. In addition, antibodies directed against SOST stimulate bone formation and represent a novel therapeutic option in the anabolic treatment of osteoporotic conditions (6,7). Mirza et al (8) demonstrated that serum SOST levels were inversely associated with estrogen levels (8). Kim et al (9) demonstrated that estrogen signaling functions as an negative regulator of SOST expression involving the $\mathrm{Wnt} / \beta$-catenin/estrogen receptor $\alpha$ $(\mathrm{ER} \alpha)$ pathway in human osteoblasts.

Icariin, extracted from Herba epimedii, has been suggested to significantly improve osteogenesis to decrease bone loss in ovariectomized rats and steroid-induced osteonecrosis of the femoral head (10). However, recent studies have demonstrated that icariin is enzymatically hydrolyzed, and then subsequently metabolized to desmethyl icaritin and icaritin; desmethyl icaritin is suggested to have more potent pharmacological effects compared with icariin $(11,12)$. Icaritin is able to promote the proliferation and differentiation of osteoblasts and enhance matrix calcification due to its estrogen-like activity (13). In addition, icaritin may decrease the activity of osteoclasts in vitro and increase the expression of osteogenic-associated mRNA levels in human BMSCs (hBMSCs) (14). The osteogenic effects of icaritin have been clearly described, but the underlying mechanisms 
remain unclear. As icaritin has been suggested to regulate the Wnt/ $\beta$-catenin pathway (10) and ER $\alpha$ (9), which are closely associated with $S O S T$, we hypothesized that icaritin may promote osteogenesis through inhibiting SOST.

The present study assessed the effects of icaritin on osteogenesis of hBMSCs and explored the role of SOST in icaritin-induced osteogenesis of hBMSCs.

\section{Materials and methods}

Reagents. Icaritin (>98\% purity) was purchased from Shanghai U-sea Biotech Co. Ltd. modified Eagle's medium of alpha $(\alpha-M E M)$ and fetal bovine serum (FBS) were both obtained from Hyclone (Hyclone; GE Healthcare Life Sciences); The Cell Counting Kit-8 (CCK-8) was purchased from Dojindo Molecular Technologies, Inc. Dimethyl sulfoxide (DMSO), trypsin and TRIzol ${ }^{\circledR}$ reagent were purchased from Gibco; Thermo Fisher Scientific, Inc.; the alkaline phosphatase (ALP) activity kit, enhanced chemiluminescent detection reagent and micro-BCA assay kit were obtained from Beyotime Institute of Biotechnology. DKK-1 and ICI182780 were purchased from R\&D Systems, Inc. The RNA extraction kit was purchased from Takara Bio, Inc. Reverse transcription-quantitative polymerase chain reaction (RT-qPCR) primers were obtained from Invitrogen; Thermo Fisher Scientific, Inc. The antibodies used were obtained from Cell Signaling Technology, Inc., unless otherwise indicated. Other reagents used in the experiment were purchased from Sigma-Aldrich; Merck KGaA. Icaritin was dissolved in DMSO and the final concentration of DMSO was $0.05 \%$ (v/v). ICI 182780 was dissolved in DMSO and stored at $4^{\circ} \mathrm{C}$. Cells were pretreated with ICI $182780(1 \mu \mathrm{M})$ for $30 \mathrm{~min}$ at $37^{\circ} \mathrm{C}$, followed by icaritin treatment.

Cell culture of hBMSCs and SOST transfection. hBMSCs were purchased from Cyagen Biosciences, Inc. (cat. no., HUXMA-01001). The hBMSCs were cultured in $\alpha$-MEM containing $10 \%$ FBS and $1 \%$ penicillin-streptomycin in a humidified incubator with $5 \% \mathrm{CO}_{2}$ at $37^{\circ} \mathrm{C}$. After reaching $80-90 \%$ confluence, the adherent cells were harvested with trypsin for $1 \mathrm{~min}$ and sub-cultured at a ratio of 1:2. Cells from passages 3-6 were used in the following experiments.

The SOST gene overexpression lentivirus was constructed and purchased from Shanghai GeneChem Co., Ltd. Briefly, the SOST gene was amplified using PCR with the following primers: SOST forward, CACCGCTGCACT TCACCC GCTACGTTTCAAGAGAACGTAGCGGGTGAAGTGCAG CTTTTTTG; and SOST reverse, GATCCAAAAAAGCTG CACTTCACCCGCTACGTTCTCTTGAAACGTAGCGGG TGAAGTGCAGC. The gene was then cloned into plenti-U bcP-IKZF2-V2-3xHA-pGK-Pur plasmid (Addgene; plasmid no. 107393) by in vitro recombination. Lentiviruses were generated by transient transfection of 293FT packaging cells (Invitrogen; Thermo Fisher Scientific, Inc.) using the calcium phosphate method. After $72 \mathrm{~h}$ of transfection, the supernatant was collected. The supernatant was filtered with a $0.45 \mu \mathrm{m}$ syringe filter (Sigma-Aldrich; cat. no. CLS431220), centrifuged for $90 \mathrm{~min}$ in at $4^{\circ} \mathrm{C}$ at $42,000 \mathrm{x} \mathrm{g}$, and resuspended in $200 \mu \mathrm{l} \alpha$-MEM. For lentivirus transduction, hBMSCs from the third passage were seeded in a 6 -well plate $\left(5 \times 10^{6}\right.$ cells/well). After $24 \mathrm{~h}$, the lentivirus solution was added to each well in serum-free Opti-MEM ${ }^{\circledR}$ (Invitrogen; Thermo Fisher Scientific, Inc.). The medium was replaced with fresh complete medium after $8 \mathrm{~h}$. The cells were selected with puromycin $(10 \mu \mathrm{g} / \mathrm{ml})$ following transduction for $48 \mathrm{~h}$. Transduction efficiency was examined by PCR and western blot analysis. The transduced cells were termed BMSCs-vector (lentiviral vector only) and BMSCs-SOST (lentiviral vector containing the SOST gene).

Identification of hBMSCs. An hBMSC suspension of $1 \times 10^{6}$ cells $/ \mathrm{ml}$ was prepared. The cells were washed twice with cold PBS, centrifuged at $1,000 \mathrm{x} \mathrm{g}$ for $5 \mathrm{~min}$ at $4^{\circ} \mathrm{C}$ and resuspended in $100 \mathrm{ml}$ stain buffer (BD Biosciences). The resuspended cells were incubated with phycoerythrin (PE)-labeled primary antibodies against surface markers integrin- $\beta 1$ (CD29; cat. no. 34971T; 1:400) and hematopoietic progenitor cell antigen CD34 (CD34; cat. no. 3569S; 1:400), as well as a corresponding isotype control antibody, at room temperature according to the manufacturers' protocol. The positively stained cells were immediately analyzed by flow cytometry using FlowJo software 8.7.1 (FlowJo, LLC). hBMSCs from passages 3-6 were used in the experiments.

Cell proliferation assay. To examine the effects of icaritin on hBMSC proliferation, the cells were seeded into a 96-well plate (5000 cells/well). The medium was removed after $24 \mathrm{~h}$, then the cells were treated with complete medium with or without different concentrations of icaritin $(0.01,0.1,1$ and $10 \mu \mathrm{M})$ accordingly for 4 days. The cells were treated with $10 \%$ CCK-8 in $100 \mu \mathrm{l}$ complete medium for $40 \mathrm{~min}$ at $37^{\circ} \mathrm{C}$ each day. The absorbance was determined at $450 \mathrm{~nm}$ using a microplate reader (ELX808; BioTek Instruments, Inc.). Cell proliferative rate (\%) was calculated as: Optical density (OD) treatment/OD control x 100 .

Osteogenic differentiation and ALP/Alizarin Red S staining. hBMSCs were seeded onto a 24 -well plate $\left(10^{5}\right.$ cells/well $)$ and cultured in osteogenic induction medium (OIM; Cyagen Biosciences, Inc.), consisting of $1 \mathrm{nM}$ dexamethasone, $50 \mathrm{mM}$ L-ascorbic acid-2-phosphate and $20 \mathrm{mM} \beta$-glycerolphosphate in complete medium, with various doses of icaritin $(0.01,0.1$ and $1 \mu \mathrm{M}$ ) for 21 days. The OIM was changed every 3 days. ALP staining and Alizarin Red S staining were conducted 7 and 14 days following the induction, respectively, and were performed separately to evaluate the positive rates of ALP and calcium deposit formation. For ALP staining, cells were fixed with $10 \%$ formaldehyde for $15 \mathrm{~min}$, rinsed three times with deionized water, and treated with the $\mathrm{BCIP}^{\circledR} / \mathrm{NBT}$ solution (Sigma-Aldrich; Merck KGaA) for 20 min. After an additional washing, the stained cultures were photographed (magnification, x10). To measure ALP activity, cell lysates were tested using a commercial ALP assay kit. For Alizarin red S staining, hBMSCs were stained with $\mathrm{pH} 4.2,0.1 \%$ Alizarin red $\mathrm{S}$ (Aladdin Industries Corporation) for $5 \mathrm{~min}$ and the images were captured using a scanner. The calcium deposition was dissolved in 10 cetylpyridinium chloride (Sigma-Aldrich; Merck KGaA), and the absorbance of the extracts was determined at $570 \mathrm{~nm}$.

$R T$ - $q P C R$. Cellular RNA was extracted from the hBMSCs cultured in OIM with or without icaritin using the RNA extraction kit, and cDNA was synthesized with the M-MLV 
Table I. Primer sequences used in PCR analysis.

\begin{tabular}{lll}
\hline Genes & Forward primer sequence (5'-3') & Reverse primer sequence (5'-3') \\
\hline$\beta$-actin & GTCATCCATGGCGAACTGGT & CGTCATCCATGGCGAACTGG \\
ALP & CAAGGATGCTGGGAAGTCCG & CTCTGGGCGCATCTCATTGT \\
Run 2 & GCTTCATTCGCCTCACAAAC & GTAGTGACCTGCGGAGATTAAC \\
$\beta$ - atenin & AAATAGCCCTGGCAGATTCC & CAGCCTCCAGCACTGTTTAT \\
SOST & CTTCACCTGACAGATCCAAGTC & CCTTCCATCCCTTCCTGTTTAG \\
\end{tabular}

ALP, alkaline phosphatase; Runx2, RUNX family transcription factor 2; OCN, osteocalcin; SOST, sclerostin.

reverse transcriptase according to the manufacturer's protocols. qPCR was conducted using Power SYBR ${ }^{\circledR}$ Green PCR Master Mix on the ABI StepOnePlus System (Applied Biosystems; Thermo Fisher Scientific, Inc.). The thermocycler conditions were as follows: Initial denaturation at $95^{\circ} \mathrm{C}$ for $5 \mathrm{~min} ; 40$ cycles at $95^{\circ} \mathrm{C}$ for $15 \mathrm{sec}$ and $60^{\circ} \mathrm{C}$ for $1 \mathrm{~min}$; and a final extension at $72^{\circ} \mathrm{C}$ for $5 \mathrm{~min}$. The primers are listed in Table I. The target gene expression was calculated using the $2^{-\Delta \Delta \mathrm{Cq}}$ method (15), and were normalized using the expression level of $\beta$-actin.

Protein extraction and western blot analysis. Cell samples were rinsed twice with cold PBS and harvested in the extraction buffer (cat. no. P0013; Beyotime Institute of Biotechnology). The lysate was centrifuged at $4^{\circ} \mathrm{C}$ with $16,000 \mathrm{x} g$ for $30 \mathrm{~min}$ and the suspension was collected. Then, the protein content was examined using a BCA kit. Total proteins $(20 \mu \mathrm{g})$ were separated by $12 \%$ (w/v) SDS-PAGE. The proteins were then transferred onto a polyvinylidene fluoride (PVDF) membrane. The membrane was washed and blocked with freshly prepared TBST containing $5 \%(\mathrm{w} / \mathrm{v})$ non-fat dry milk for $90 \mathrm{~min}$ at room temperature. The membrane was incubated with antibodies targeting $\beta$-actin (1:1,000; cat. no. 4970S; Cell Signaling Technology, Inc.), osteocalcin (OCN; 1:1,000; cat. no. MAB1419; R\&D Systems, Inc.), RUNX family transcription factor 2 (Runx2; 1:1,000; cat. no. 12556; Cell Signaling Technology, Inc.), ALP (1:1,000; cat. no. AF2910; R\&D Systems, Inc.), $\beta$-catenin $(1: 1,000$; cat. no. 8480S; Cell Signaling Technology, Inc.) and SOST (1:1,000; cat. no. MAB1406; R\&D Systems, Inc.) overnight at $4^{\circ} \mathrm{C}$. Following three washes, the membrane was incubated with horseradish peroxidase-conjugated goat anti-rabbit or anti-mouse secondary antibody (1:1,000; cat. nos. 93702 and 7076S, respectively; Cell Signaling Technology, Inc.) for $1 \mathrm{~h}$ at room temperature. The membrane was washed three times and then the protein-antibody complexes were examined using an enhanced chemiluminescent detection reagent. Antibody signals were developed using a Bio-Rad XRS chemiluminescence detection system (Bio-Rad Laboratories, Inc.). Band densities were analyzed using Quantity One Software (Bio-Rad Laboratories, Inc.; v4.52). The mean expression levels of the proteins relative to $\beta$-actin were presented.

Immunofluorescence staining. Following fixing with $4 \%$ paraformaldehyde at $4^{\circ} \mathrm{C}$ for $15 \mathrm{~min}$, the hBMSCs were treated with $0.25 \%$ Triton $\mathrm{X}-100$ and $2 \%$ bovine serum albumin at $4{ }^{\circ} \mathrm{C}$ for 15 and $30 \mathrm{~min}$, respectively. hBMSCs were washed and incubated overnight with primary anti-SOST antibody (1:100; cat. no. MAB1406; R\&D Systems, Inc.) at $4^{\circ} \mathrm{C}$. After washing three times with PBS, the samples were incubated with a green fluorescence-labeled rabbit anti-mouse secondary antibody (1:100; cat. no. ab150113; Abcam) for $2 \mathrm{~h}$ in the dark at $4^{\circ} \mathrm{C}$. After the nuclei were stained with DAPI $(5 \mu \mathrm{g} / \mathrm{ml})$ for $5 \mathrm{~min}$, the cells were observed using a fluorescence microscope (magnification, x100; Leica Microsystems GbmH).

Statistical analysis. All data acquisitions were repeated 3 times and were analyzed using SPSS v.18.0 (SPSS, Inc.). A one-way analysis of variance followed by Bonferroni's post-hoc test was used for multi-group comparison, and differences between two groups were determined by Student's $\mathrm{t}$-test. The data are presented as the mean \pm standard deviation. $\mathrm{P}<0.05$ was considered to indicate a statistically significant difference.

\section{Results}

Identification of human MSCs. hBMSCs at passage 3 were harvested and the surface phenotypes were assessed by flow cytometry (Fig. 1). The results showed that $94.7 \%$ of the cells expressed CD29 and only 1.3\% of the cells expressed CD34. These results suggested that the isolated hBMSCs expressed standard markers and were suitable for use in the following experiments.

Icaritin did not affect proliferation of hBMSCs. To determine whether icaritin would promote cell proliferation of hBMSCs, a CCK-8 assay was used. hBMSCs were treated with various concentrations of icaritin $(0,0.01,0.1,1$ and $10 \mu \mathrm{M})$ for 1-4 days. The results indicated that icaritin at $0.01-1 \mu \mathrm{M}$ did not cause any significant toxicity to the cells; however, icaritin started to exert toxic effects when the dose reached $10 \mu \mathrm{M}$ (Fig. 2). Therefore, concentrations of icaritin from 0.01 to $1 \mu \mathrm{M}$ were chosen for further experimentation.

Icaritin enhanced osteogenesis of hBMSCs. The data indicated that $0.01-1 \mu \mathrm{M}$ icaritin could induce the formation of calcified nodules of hBMSCs, particularly at the concentration of $1 \mu \mathrm{M}$ (Fig. 3A), which was confirmed by the results from Alizarin red S quantitative assay (Fig. 3B). ALP activity can 


\section{A}

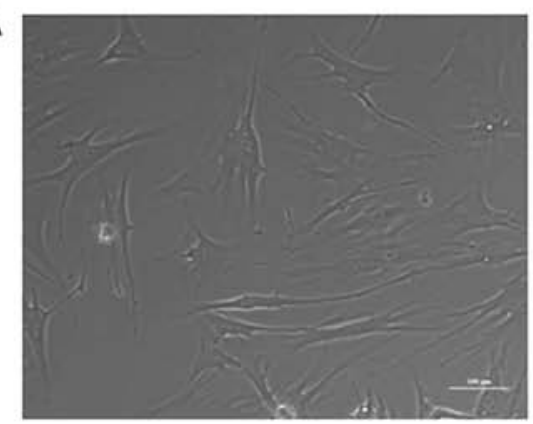

Primary cells of hBMSCs $\times 100$

B

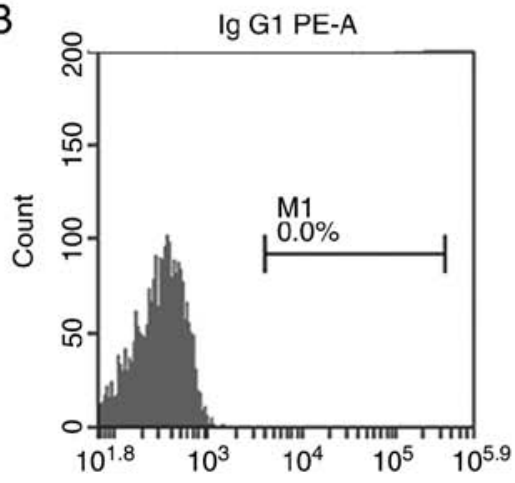

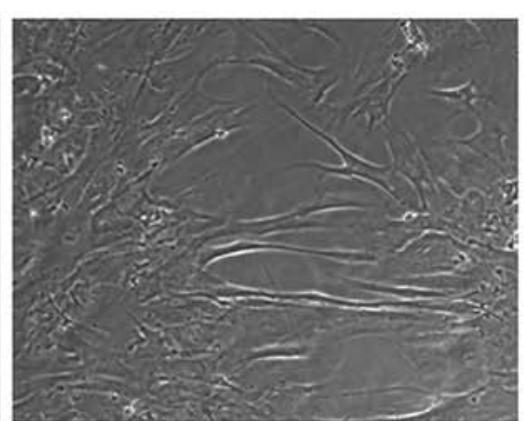

2 Passages of hBMSCs $\times 100$

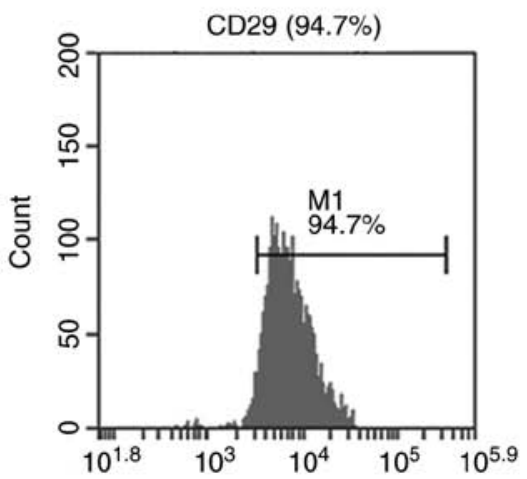

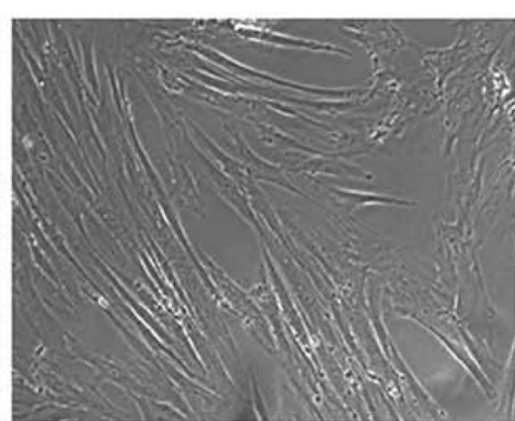

3 Passages of hBMSCs $\times 100$

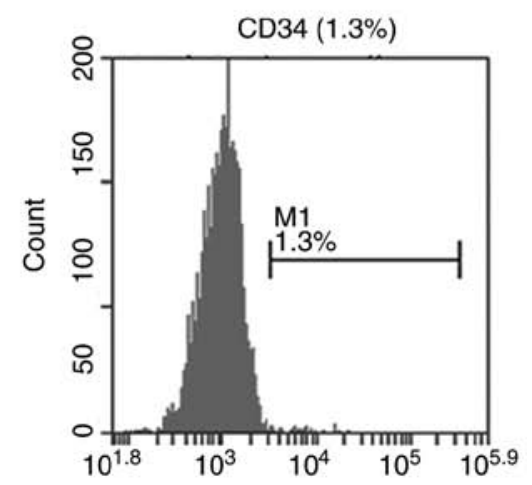

Figure 1. Characterization of hBMSCs. (A) Morphology and (B) flow cytometry analysis of hBMSCs. hBMSCs expressed CD29, but not CD34. hBMSCs, human bone marrow-derived mesenchymal stem cells; PE, phycoerythrin; CD29, integrin- $\beta 1$; CD34, hematopoietic progenitor cell antigen CD34.

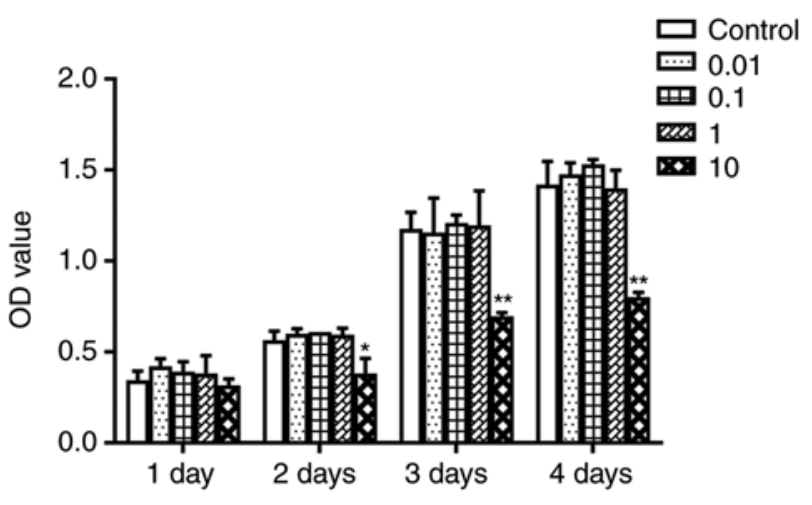

Figure 2. Effects of icaritin on hBMSC proliferation measured by CCK- 8 assay. The cells were incubated with icaritin $(0.01-10 \mu \mathrm{M})$ for $1,2,3$ and 4 days. At each time point, CCK- 8 was added to each group to detect the proliferation ability. All data are presented as mean \pm standard deviation from 3 independent experiments. ${ }^{*} \mathrm{P}<0.05$ and ${ }^{* *} \mathrm{P}<0.01$ vs. control group. hBMSCs, human bone marrow-derived mesenchymal stem cells; CCK- 8 , Cell Counting Kit-8; OD, optical density.

be used to detect the early stage of osteogenesis. Therefore, the ALP activity of BMSCs treated with or without icaritin was examined at 7 days after osteogenic induction. The results demonstrated that ALP activity in the icaritin group was increased almost 1.8-fold compared with that in the control group (Fig. 3C). RT-qPCR was also used to detect the osteogenic gene expression at 7 days. The results revealed that the levels of osteogenic genes (OCN, Runx2 and ALP) in the icaritin group were significantly increased compared with those in the control group (Fig. 3D-F). The results suggested that icaritin significantly enhanced osteogenesis of hBMSCs.
Effects of icaritin on mRNA and protein levels of osteogenic genes. The effects of icaritin on the mRNA levels of $O C N$, Runx2, ALP and $\beta$-catenin were further determined at days 3, 7 and 14. The RT-qPCR results suggested that icaritin upregulated $O C N$ at days 7 and 14, and Runx2 at day 7, and increased ALP and $\beta$-catenin transcript levels at days 3 and 7 (Fig. 4A-D). However, icaritin decreased the expression level of SOST at days 7 and 14 (Fig. 4E). In addition, the data from the western blot analysis indicated that icaritin exhibit similar effects on the expression levels of osteogenic proteins as with the mRNA levels (Fig. 5).

Lentivirus-mediated SOST overexpression reverses the effects of icaritin on osteogenic differentiation. To investigate SOST function, hBMSCs were transfected with lentiviruses encoded with the SOST gene to overexpress SOST. The mRNA and protein expression levels of SOST were significantly increased in the SOST overexpression group compared with in the vector control group, as determined by RT-qPCR and western blot analysis. Furthermore, overexpression of SOST partly inhibited the icaritin-induced increase of ARS level, ALP activity and osteogenic gene expression. As demonstrated in Fig. 6, the ARS level and ALP activity in the BMSCs-vector + icaritin group were significantly increased compared with that in the BMSCs-SOST + icaritin group. In addition, the BMSCs-SOST + icaritin group also exhibited decreased levels of $\beta$-catenin and osteogenic genes including $O C N$, Runx 2 and $A L P$.

Icaritin suppresses SOST protein expression mediated by the Wnt/ER $\alpha$ signaling pathway in hBMSCs. To examine whether the Wnt/ER $\alpha$ signaling pathway was involved in icaritin-induced suppression of SOST, the protein level of 

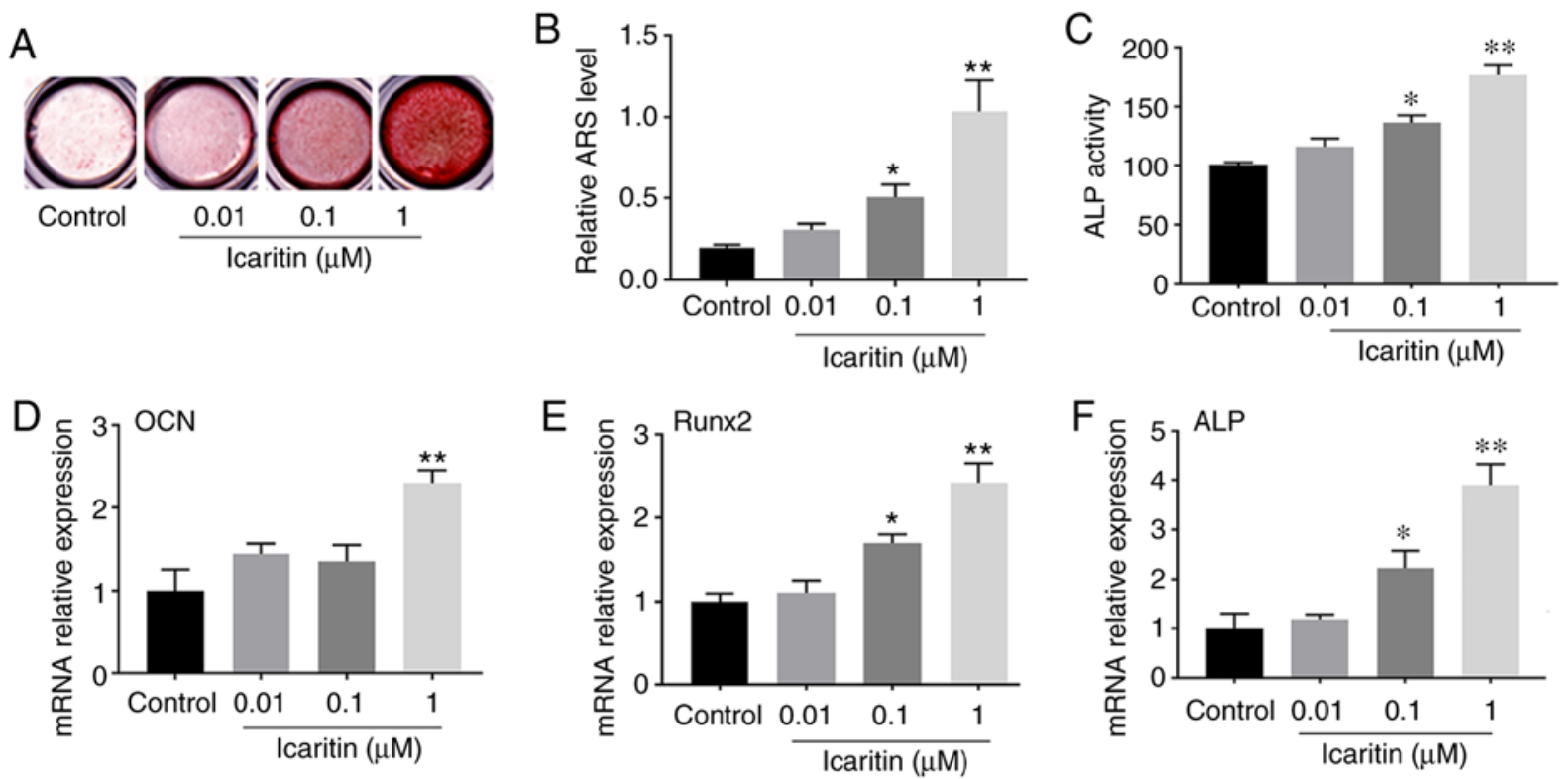

Figure 3. Icaritin promotes osteogenesis on hBMSCs. (A) Alizarin Red S stainingto visualize mineralization was applied following treatment of the hBMSCs with icaritin at various concentrations (0.01-1 $\mu \mathrm{M})$ for 21 days. Magnification, x10. (B) The mineralization of hBMSCs of Alizarin red S was quantified. (C) ALP activity of hBMSCs treated with icaritin for 7 days. (D-F) hBMSCs were treated with Icaritin for 3 days, and the mRNA levels of (D) OCN, (E) Runx2 and (F) Alp were determined by reverse transcription quantitative polymerase chain reaction. Data are presented as mean \pm standard deviation ( $\mathrm{n}=3$ ). ${ }^{*} \mathrm{P}<0.05$ and ${ }^{* *} \mathrm{P}<0.01$ vs. control group. hBMSCs, human bone marrow-derived mesenchymal stem cells; ALP; OCN, osteocalcin; Runx2, RUNX family transcription factor 2; Alp, alkaline phosphatase.
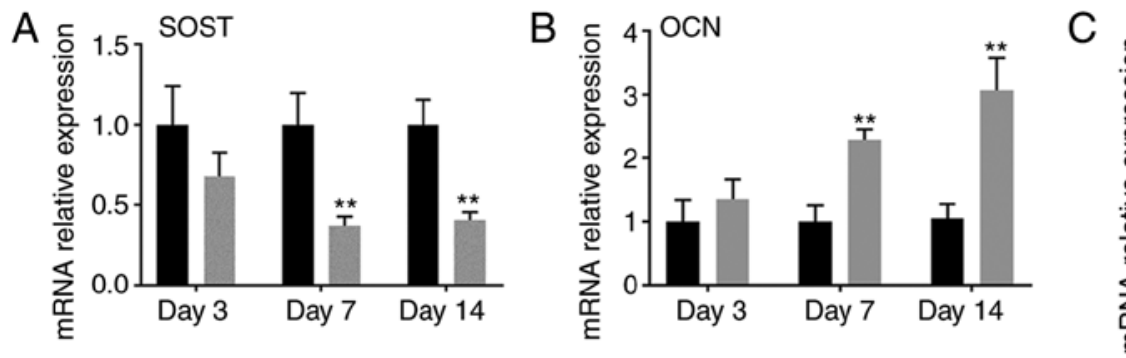

$\mathrm{E}$

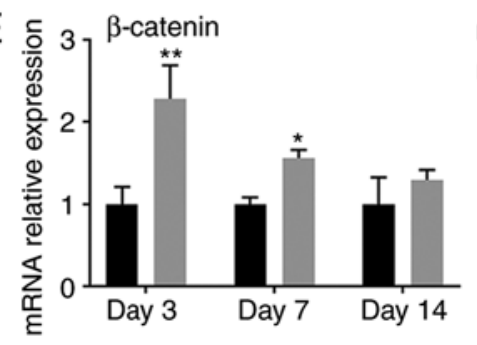

Figure 4. Icaritin $(1 \mu \mathrm{M})$ increases mRNA levels of osteogenic marker genes. (A) SOST, (B) OCN, (C) Runx2, (D) Alp and (E) $\beta$-catenin at the different time points of osteogenesis of human bone marrow-derived mesenchymal stem cells. DMSO was used as the control group. Data are presented as the mean \pm standard deviation $(\mathrm{n}=3)$. ${ }^{*} \mathrm{P}<0.05$ and ${ }^{* *} \mathrm{P}<0.01$ vs. control group at the same stage. SOST, sclerostin; OCN, osteocalcin; Runx 2 , RUNX family transcription factor 2; Alp, alkaline phosphatase.

SOST in hBMSCs treated with icaritin and the Wnt inhibitor, ICI 182,780 , which causes ER $\alpha$ degradation, were detected by western blot analysis at days 3, 7 and 14 . Icaritin decreased the protein expression of SOST significantly at day 3, while the decrease was not that marked at days 7 and 14. ICI 182,780 treatment partially reversed the suppression of SOST caused by icaritin, to levels close to those of the control group. The results of the present study indicated that icaritin decreased SOST expression and was regulated via the Wnt/ER $\alpha$ signaling pathway (Fig. 7A and B). To confirm these data, a immunofluorescence assay was conducted; a decreased SOST (green) fluorescence signal in the cells treated with icaritin was observed, and pretreatment with ICI 182780 was demonstrated to reverse the process (Fig. 8).

\section{Discussion}

The results of the present study demonstrated that the small phytomolecule icaritin enhanced osteogenic differentiation of hBMSCs in vitro, with the greatest effect demonstrated 
A

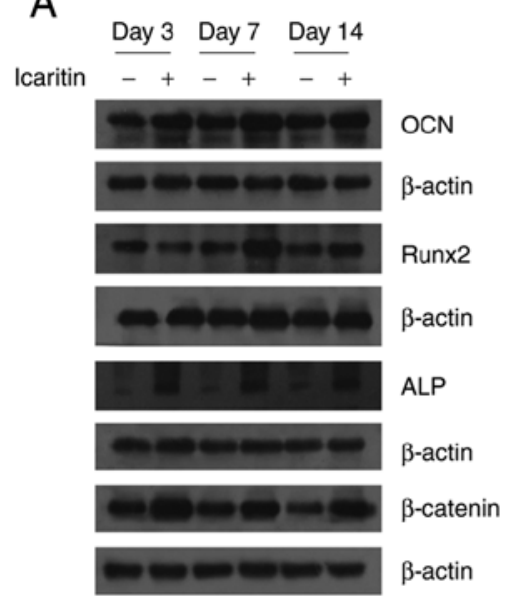

B
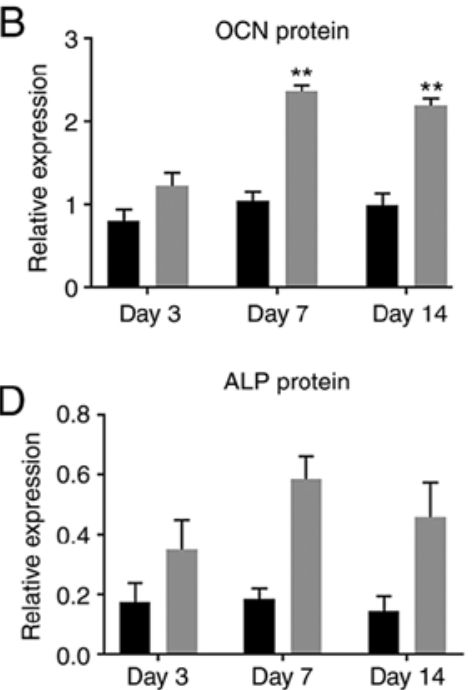

C
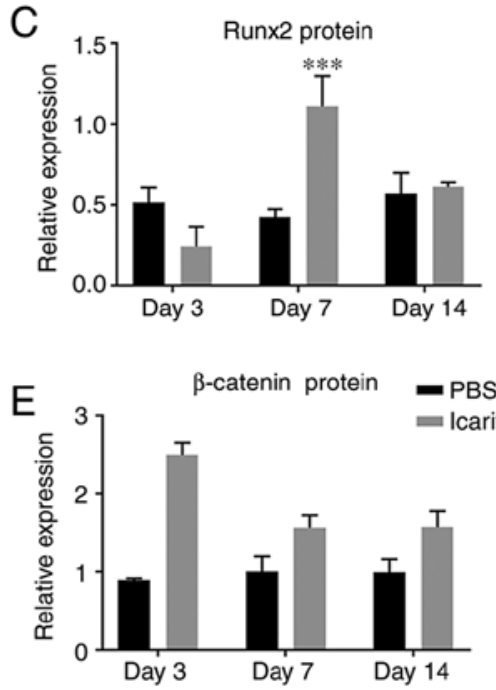

Figure 5. Icaritin $(1 \mu \mathrm{M})$ increases protein levels of osteogenic genes. (A) Human bone marrow-derived mesenchymal stem cells were cultured in OIM for 3, 7, 14 days, and OCN, Runx2, ALP, $\beta$-catenin protein levels were measured. DMSO was used as control group. (B-E) Band density of (B) OCN, (C) Runx2, (D) ALP and (E) $\beta$-catenin were quantified by densitometry. Data are presented as mean \pm standard deviation $(\mathrm{n}=3) .{ }^{* *} \mathrm{P}<0.01,{ }^{* * *} \mathrm{P}<0.001$ vs. control group at the same day. OCN, osteocalcin; Runx2, RUNX family transcription factor 2; Alp, alkaline phosphatase.

A

$$
\begin{array}{r}
\text { Sclerostin } \\
\beta \text {-actin }
\end{array}
$$

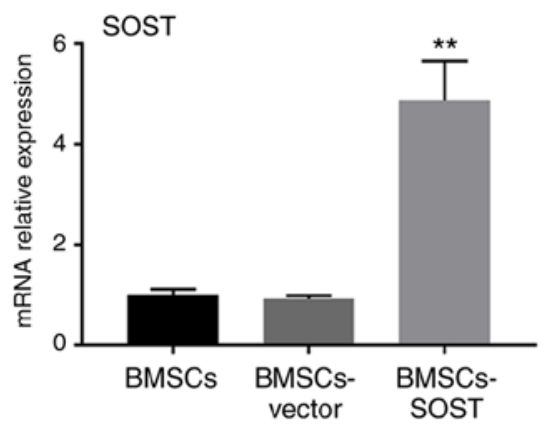

C
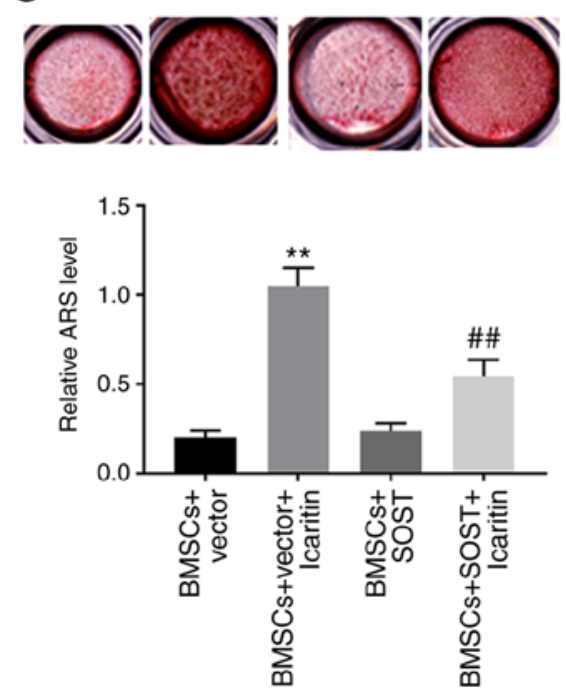

$\mathrm{B}$
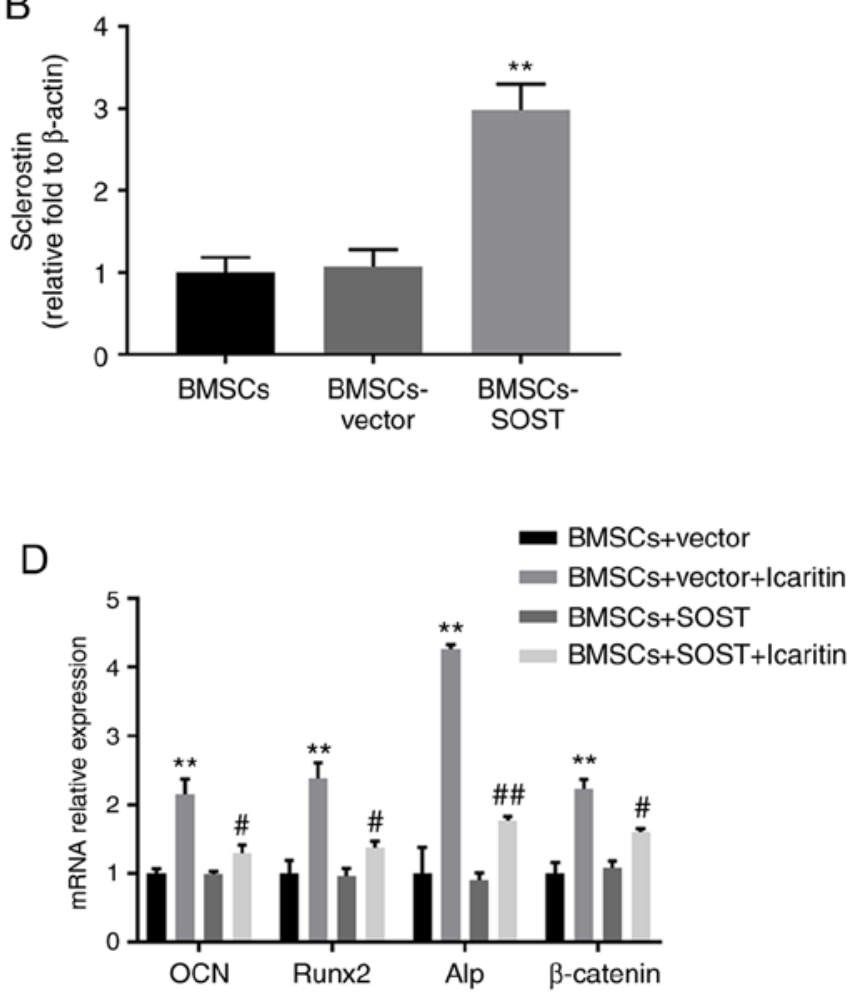

Figure 6. SOST overexpression reverses icaritin-induced osteogenesis of hBMSCs. (A) Western blot analysis for the protein level of SOST. (B) The mRNA expression of SOST in BMSCs, BMSCs-vector, and BMSCs-SOST. (C) Mineralization in cultured hBMSCs in BMSCs-vector and BMSCs-SOST groups with or without icaritin were detected at day 14. Magnification, x10. (D) The mRNA levels of OCN, Runx2, Alp and $\beta$-actin were determined by reverse transcription quantitative polymerase chain reaction. Data are presented as mean \pm standard deviation $(\mathrm{n}=3)$. ${ }^{* *} \mathrm{P}<0.01$ vs. BMSCs group; ${ }^{"} \mathrm{P}<0.05$ and ${ }^{\# \#} \mathrm{P}<0.01$ vs. BMSCs-vector group. SOST, sclerostin; BMSCs, bone marrow-derived mesenchymal stem cells; OCN, osteocalcin; Runx2, RUNX family transcription factor 2; Alp, alkaline phosphatase. 
A
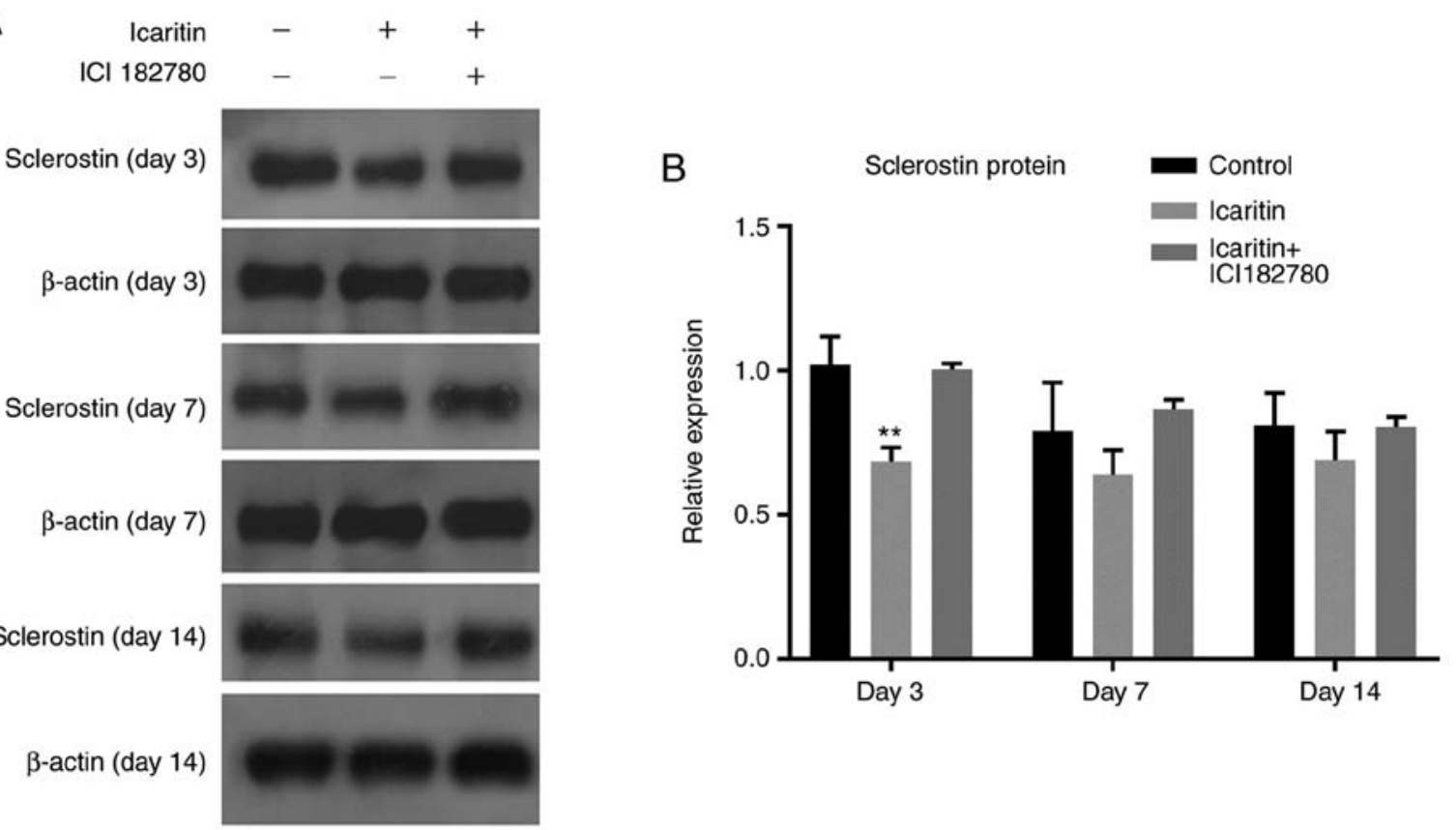

Figure 7. Icaritin downregulates SOST, which is reversed by ICI 182780. hBMSCs were cultured in osteogenic induction medium for 3 , 7 and 14 days, and SOST protein levels were measured. DMSO was served as the control group. (A) SOST protein expression was measured by western blot analysis. (B) Band density of SOST was quantified by densitometry. Data are presented as mean \pm standard deviation $(\mathrm{n}=3)$. $^{* *} \mathrm{P}<0.01$ vs. control group at the same day. SOST, sclerostin; BMSCs, bone marrow-derived mesenchymal stem cells; OCN, osteocalcin; Runx2, RUNX family transcription factor 2; Alp, alkaline phosphatase.

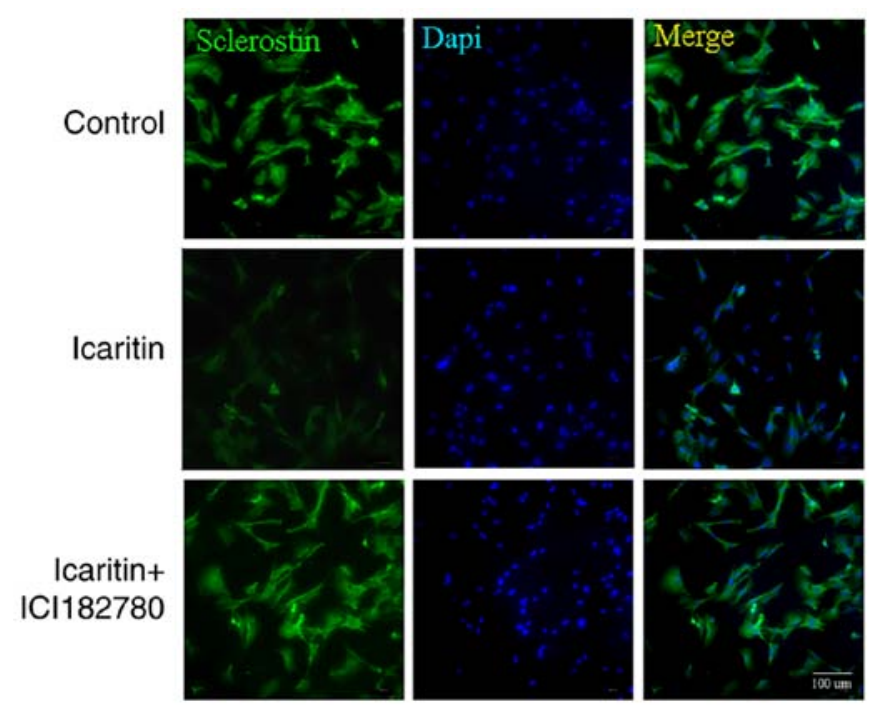

Figure 8. Effects of icaritin + ICI 182780 on the expression of sclerostin detected by immunofluorescence staining. hBMSCs were divided into three groups: Icaritin; icaritin + ICI 182780; and DMSO. Following culture in osteogenic induction medium for 14 days, all the groups were collected for immunofluorescence staining. Sclerostin was labeled with green fluorescence and the nuclei were stained with DAPI.

at $1 \mu \mathrm{M}$ concentration. The results also demonstrated that SOST served a crucial role in icaritin-induced osteogenesis of hBMSCs. Icaritin downregulated SOST expression at mRNA and protein level, as demonstrated by the RT-qPCR and western blot analysis. Overexpression of SOST inhibited icaritin-induced osteogenic differentiation. It was also identified that SOST expression was mediated through icaritin-induced activation of the Wnt/ $\beta$-catenin and ER $\alpha$ signaling pathways.
These results suggest that SOST is a key factor in the icaritin-mediated osteogenesis of hBMSCs.

Herba epimedii is used for curing bone-associated disorders in China (10). Icariin is considered to be the major pharmacologically active component and it promotes osteogenesis of hBMSCs (10). Recent studies have revealed that the pharmacological effects of icaritin, which is an intestinal metabolism of icariin, were more potent than icariin in vitro $(11,12)$. In the present study, it was first demonstrated icaritin had no effects on the proliferative ability or viability of hBMSCs at a wide range of concentrations $(0.01-1 \mu \mathrm{M})$. The dose would be much lower if used in vivo, suggesting that icaritin may achieve a bio-safety level for clinical use. Whether icaritin enhanced osteogenesis of BMSCs was further explored through the detection of ALP activity and calcium nodule formation. The results demonstrated that icaritin promoted both of these osteoblast markers. Next, the effects of icaritin on osteogenic marker genes of hBMSCs were examined. It was identified that the levels of ALP, Runx 2 and OCN were elevated following icaritin treatment. ALP, an early marker of osteogenesis, is a key regulator in bone formation (16), and is able to produce phosphate, which reacts with calcium to form hydroxyapatite, further promoting mineralization. Runx 2 , a pivotal transcription regulator, is the runt family transcription factor, which serves an important role in osteogenesis $(17,18) . O C N$ is a marker gene of late stage osteoblast differentiation (17). Icaritin significantly increased the mRNA and protein levels of these osteogenic marker genes, suggesting that icaritin promoted osteogenesis of hBMSCs.

The Wnt/ $\beta$-catenin pathway is well-known as a key axis for modulating bone mass (13). SOST, the Wnt/ $\beta$-catenin regulator, inhibits bone formation by exerting antagonistic effects 
on Wnt pathway. Antagonism or loss of the SOST will result in elevated bone formation and a high bone mass $(19,20)$. The SOST antibody (SclAb) has attracted attention as an anabolic strategy for treating post-menopausal osteoporosis (21). Furthermore, in phase II (ClinicalTrials.gov identifier: NCT01059435) (22) and III clinical trials (ClinicalTrials. gov identifier: NCT01575834) (23), SclAb increased bone mass and strength in osteoporotic women. The results of the present study indicated that icaritin downregulated the mRNA and protein levels of SOST and upregulated the mRNA level of $\beta$-catenin. When SOST was overexpressed in hBMSCs, the mRNA level of $\beta$-catenin decreased, which is consistent with previous data indicating that SOST antagonizes the Wnt signaling pathway (9). To the best of our knowledge, the present study demonstrated for the first time that overexpression of SOST diminishes the icaritin-induced increases in ALP activity, ARS level and osteogenic genes.

To determine whether the Wnt/ER $\alpha$ signaling pathway was involved in icaritin-induced suppression of SOST, hBMSCs were treated with icaritin and the Wnt inhibitor, ICI 182,780, which causes ER $\alpha$ degradation (24). The results demonstrated that icaritin treatment decreased the levels of SOST protein expression, while treatment with ICI 182,780 partly reversed the icaritin-mediated suppression of SOST, indicating that the Wnt/ $\beta$-catenin and ER $\alpha$ signaling pathways were involved in icaritin-induced SOST suppression. These results were consistent with previous data suggesting that estrogen-mediated suppression of SOST expression was associated with the $\mathrm{Wnt} / \beta$-catenin/ER $\alpha$ axis in human osteoblasts (24).

Stem cell transplantation has been applied in clinical settings for the treatment of multiple conditions, such as diabetic neuropathy (25) and leukemia (26). However, stem cell transplantation alone has exhibited limited satisfactory results Previously, the combination of stem cells with other compounds with osteogenic effects may provide improved therapeutic effects. Icariin, which has been suggested to exhibit weaker pharmacological effects (12) was demonstrated to result in significantly decreased levels of bone loss in postmenopausal women (27). Based on these data, we hypothesized that icaritin, which had significant osteogenic effects, may also be used for decreasing the bone loss in postmenopausal women.

In conclusion, icaritin induced osteogenesis in hBMSCs by suppressing SOST expression, and icaritin-induced suppression of SOST was regulated in part via the Wnt/ $\beta$-catenin and $\mathrm{ER} \alpha$ pathways. The results of the present study suggested that icaritin may be a promising agent for diseases caused by decreased levels of osteogenic differentiation of BMSCs, such as osteoporosis or osteonecrosis of the femoral head.

\section{Acknowledgements}

We are thankful to Dr Liangliang Xu for providing technical suggestions for the experiments.

\section{Funding}

The present study was supported by grants from the project of the National Natural Science Foundation of China (grant nos. 8187031444 and 81574002 ).

\section{Availability of data and materials}

All the materials included in the manuscript, including all relevant raw data, may be made freely available to any researchers who wish to use them for non-commercial purposes, while preserving any necessary confidentiality and anonymity.

\section{Authors' contributions}

$\mathrm{YC}$ conceived and supervised the project. BW and $\mathrm{HH}$ were in charge of the PCR, western blotting, flow cytometry and immunofluorescence staining. QW was responsible for the experimental design, data analysis, drafting and revision of the article. CX analyzed data. LL and JG were responsible for the culture of the hBMSCs, ALP/Alizarin Red S staining, CCK-8 assay and lentiviral transduction. All authors read and approved the final manuscript.

\section{Ethics approval and consent to participate}

Not applicable.

\section{Patient consent for publication}

Not applicable.

\section{Competing interests}

The authors declare that they have no competing interests.

\section{References}

1. Liu C, Zhang H, Tang X, Feng R, Yao G, Chen W, Li W, Liang J, Feng $X$ and Sun L: Mesenchymal stem cells promote the osteogenesis in collagen-induced arthritic mice through the inhibition of TNF- $\alpha$. Stem Cells Int 2018: 4069032, 2018.

2. Huang Z, Cheng C, Cao B, Wang J, Wei H, Liu X, Han Y, Yang S and Wang $X$ : Icariin protects against glucocorticoid-induced osteonecrosis of the femoral head in rats. Cell Physiol Biochem 47: 694-706, 2018.

3. Wei Q, He M, Chen M, Chen Z, Yang F, Wang H, Zhang J and $\mathrm{He} \mathrm{W}$ : Icariin stimulates osteogenic differentiation of rat bone marrow stromal stem cells by increasing TAZ expression. Biomed Pharmacother 91: 581-589, 2017.

4. Zhang D, Park BM, Kang M, Nam H, Kim EJ, Bae C and Lim SK: The systemic effects of sclerostin overexpression using $\Phi C 31$ integrase in mice. Biochem Biophys Res Commun 472: 471-476, 2016.

5. Pietrzyk B, Smertka M and Chudek J: Sclerostin: Intracellular mechanisms of action and its role in the pathogenesis of skeletal and vascular disorders. Adv Clin Exp Med 26: 1283-1291, 2017.

6. McDonald MM, Morse A, Birke O, Yu NYC, Mikulec K, Peacock L, Schindeler A, Liu M, Ke HZ and Little DG: Sclerostin antibody enhances bone formation in a rat model of distraction osteogenesis. J Orthop Res 36: 1106-1113, 2018.

7. McClung MR: Sclerostin antibodies in osteoporosis: Latest evidence and therapeutic potential. Ther Adv Musculoskelet Dis 9: 263-270, 2017.

8. Mirza FS, Padhi ID, Raisz LG and Lorenzo JA: Serum sclerostin levels negatively correlate with parathyroid hormone levels and free estrogen index in postmenopausal women. J Clin Endocrinol Metab 95: 1991-1997, 2010.

9. Kim RY, Yang HJ, Song YM, Kim IS and Hwang SJ: Estrogen modulates bone morphogenetic protein-induced sclerostin expression through the Wnt signaling pathway. Tissue Eng Part A 21: 2076-2088, 2015.

10. Huang JM, Bao Y, Xiang W, Jing XZ, Guo JC, Yao XD, Wang R and Guo FJ: Icariin regulates the bidirectional differentiation of bone marrow mesenchymal stem cells through canonical Wnt signaling pathway. Evid Based Complement Alternat Med 2017: 8085325, 2017. 
11. Wu T, Shu T, Kang L, Wu J, Xing J, Lu Z, Chen S and Lv J: Icaritin, a novel plant-derived osteoinductive agent, enhances the osteogenic differentiation of human bone marrow- and human adipose tissue-derived mesenchymal stem cells. Int J Mol Med 39: 984-992, 2017.

12. Wang ZQ and Lou YJ: Proliferation-stimulating effects of icaritin and desmethylicaritin in MCF-7 cells. Eur J Pharmacol 504: 147-153, 2004.

13. Baron R and Gori F: Targeting WNT signaling in the treatment of osteoporosis. Curr Opin Pharmacol 40: 134-141, 2018.

14. Sheng H, Rui XF, Sheng CJ, Li WJ, Cheng XY, Jhummon NP, Yu YC, Qu S, Zhang G and Qin L: A novel semisynthetic molecule icaritin stimulates osteogenic differentiation and inhibits adipogenesis of mesenchymal stem cells. Int J Med Sci 10 : 782-789, 2013

15. Livak KJ and Schmittgen TD: Analysis of relative gene expression data using real-time quantitative PCR and the 2(-Delta Delta C(T)) method. Methods 25: 402-408, 2001

16. Harrison G, Shapiro IM and Golub EE: The phosphatidylinositol-glycolipid anchor on alkaline phosphatase facilitates mineralization initiation in vitro. J Bone Miner Res 10: 568-573, 1995.

17. Orimo $\mathrm{H}$ : The mechanism of mineralization and the role of alkaline phosphatase in health and disease. J Nippon Med Sch 77: $4-12,2010$.

18. Zhao Z, Zhao M, Xiao G and Franceschi RT: Gene transfer of the Runx 2 transcription factor enhances osteogenic activity of bone marrow stromal cells in vitro and in vivo. Mol Ther 12 : 247-253, 2005

19. Taylor S, Hu R, Pacheco E, Locher K, Pyrah I, Ominsky MS and Boyce RW: Differential time-dependent transcriptional changes in the osteoblast lineage in cortical bone associated with sclerostin antibody treatment in ovariectomized rats. Bone Rep 8: 95-103, 2018.

20. Zhao W, Li X, Peng Y, Qin Y, Pan J, Li J, Xu A, Ominsky MS Cardozo C, Feng JQ, et al: Sclerostin antibody reverses the severe sublesional bone loss in rats after chronic spinal cord injury. Calcif Tissue Int 103: 443-454, 2018.
21. Bhattacharyya S,Pal S and Chattopadhyay N: Targeted inhibition of sclerostin for post-menopausal osteoporosis therapy: A critical assessment of the mechanism of action. Eur J Pharmacol 826: 39-47, 2018.

22. McClung MR, Grauer A, Boonen S, Bolognese MA, Brown JP, Diez-Perez A, Langdahl BL, Reginster JY, Zanchetta JR, Wasserman SM, et al: Romosozumab in postmenopausal women with low bone mineral density. N Engl J Med 370: 412-420, 2014

23. Lewiecki EM: Role of sclerostin in bone and cartilage and its potential as a therapeutic target in bone diseases. Ther Adv Musculoskelet Dis 6: 48-57, 2014.

24. Mbalaviele G, Sheikh S, Stains JP, Salazar VS, Cheng SL, Chen D and Civitelli R: Beta-catenin and BMP-2 synergize to promote osteoblast differentiation and new bone formation. J Cell Biochem 94: 403-418, 2005.

25. Datta I, Bhadri N, Shahani P, Majumdar D, Sowmithra S, Razdan R and Bhonde R: Functional recovery upon human dental pulp stem cell transplantation in a diabetic neuropathy rat model. Cytotherapy 19: 1208-1224, 2017.

26. Heidrich K, Thiede C, Schäfer-Eckart K, Schmitz N,Aulitzky WE, Krämer A, Rösler W, Hänel M, Einsele H, Baldus CD, et al: Allogeneic hematopoietic cell transplantation in intermediate risk acute myeloid leukemia negative for FLT3-ITD, NPM1- or biallelic CEBPA mutations. Ann Oncol 28: 2793-2798, 2017.

27. Wang Z, Wang D, Yang D, Zhen W, Zhang J and Peng S: The effect of icariin on bone metabolism and its potential clinical application. Osteoporos Int 29: 535-544, 2018.

This work is licensed under a Creative Commons Attribution-NonCommercial-NoDerivatives 4.0 International (CC BY-NC-ND 4.0) License. 